\title{
2 FATORES DE RISCO E IDEAÇÃO SUICIDA ENTRE ESTUDANTES DE ENFERMAGEM
}

\author{
| Liliane da Silva ${ }^{1}$; Alisséia Lemes²; Vagner do Nascimento ${ }^{3}$; Rosa Volpato ${ }^{4}$; Elias da Rocha ${ }^{5}$; Adaene de Moura ${ }^{6}$ |
}

\section{RESUMO}

CONTEXTO: A universidade é um local de desenvolvimento científico e humano, com alto risco ao adoecimento mental. As repercussões desse adoecimento, podem desencadear processos de questionamento sobre a vida, valores individuais e em algumas circunstâncias contribuir para a presença de ideação suicida.

OBJETIVO: Avaliar a presença de ideação suicida entre os estudantes de enfermagem e os fatores de risco associados.

MÉTODOS: Pesquisa exploratória, com abordagem quantitativa, realizada entre julho e outubro de 2016, com estudantes de enfermagem de uma universidade pública do Brasil. A coleta de dados ocorreu com aplicação de questionário semiestruturado e Inventário de Ideação suicida de Beck. Os dados foram analisados pelo Epiinfo versão 3.5.2 e Bioestat versão 5.0.

RESULTADOS: Participaram da pesquisa 60 estudantes de enfermagem, entre 18 e 37 anos, sendo $80 \%$ do sexo feminino e $82 \%$ solteiros. Em relação aos fatores de risco para o suicídio destacou-se a dificuldade financeira (33\%) e mudança de cidade para cursar a universidade (45\%). Houve associação entre ideação suicida com maiores responsabilidades (100\%) e pressão (86\%) na vida atual. Esta associação foi mais significativa entre o histórico para tentativa de suicídio $(\mathrm{p}=0,015)$ e depressão $(\mathrm{p}=0,010)$. A ideação suicida esteve presente em $12 \%$ da amostra. CONCLUSÃO: Neste estudo, verificou-se que parte dos estudantes apresentaram ideação suicida, ao mesmo tempo que estão expostos a fatores de risco que podem contribuir para desenvolver ou agravar o adoecimento mental, motivo que alerta a gestão universitária quanto ao cuidado à saúde do estudante desde o ingresso à formação.

\section{PALAVRAS-CHAVE: Estudantes de enfermagem; Prevenção; Saúde mental; Suicídio}

\section{RESUMEN}

\section{"Factores de riesgo y idenación suicida entre estudiantes de en-} fermería”

CONTEXTO: La universidad es un lugar de desarrollo científico y humano, con alto riesgo de enfermedad mental. Las repercusiones de esta enfermedad pueden desencadenar procesos de cuestionamiento sobre la vida, los valores individuales y en algunas circunstancias, contribuyen a la presencia de ideas suicidas.

OBJETIVO: Valorar la presencia de ideación suicida entre estudiantes de enfermería y los factores de riesgo asociados.

METODOLOGÍA: Investigación exploratoria con enfoque cuantitativo, realizada entre julio y octubre de 2016, con estudiantes de enfermería de una universidad pública de Brasil. La recolección de datos se realizó mediante un cuestionario semiestructurado y el inventario de ideas suicidas de Beck. Los datos fueron analizados por Epiinfo versión 3.5.2 y Bioestat versión 5.0.

RESULTADOS: Participaron de la investigación 60 estudiantes de enfermería, entre 18 y 37 años, $80 \%$ mujeres y $82 \%$ solteros. En cuanto a los factores de riesgo de suicidio, se destacaron las dificultades financieras (33\%) y el cambio de ciudad para estudiar en la universidad (45\%). Hubo una asociación entre ideación suicida con responsabilidades más altas (100\%) y presión (86\%) en la vida actual. Esta asociación fue más significativa entre la historia de intento de suicidio $(\mathrm{p}=0.015)$ y depresión $(\mathrm{p}=0.010)$. La ideación suicida estuvo presente en el $12 \%$ de la muestra.

CONCLUSIÓN: En este estudio, se descubrió que parte de los estudiantes presentaban ideas suicidas, mientras estaban expuestos a factores de riesgo que pueden contribuir a desarrollar o agravar una enfermedad mental, razón que alerta a la gerencia de la universidad sobre la atención médica del estudiante desde la entrada a la formación.

\section{DESCRIPTORES: Estudiantes de enfermería; Prevención; Salud} mental; El suicidio

\section{ABSTRACT}

\section{"Risk factors and suicide ideation among nursing students"}

BACKGROUND: The university is a place of scientific and human development, with high risk for mental illness. The repercussions of this illness can triøger processes of questioning about life, individual values, and in some circumstances contribute to the presence of suicidal ideation.

AIM: Assess the presence of suicidal ideation among nursing students and the associated risk factors.

METHODS: Exploratory research with quantitative approach, conducted between July and October 2016, with nursing students from a public university in Brazil. Data collection was performed using a semi-structured questionnaire and Beck's suicidal ideation inventory. Data were analyzed by Epiinfo version 3.5.2 and Bioestat version 5.0.

RESULTS: Sixty nursing students, between 18 and 37 years old, participated in the research, $80 \%$ female and $82 \%$ single.

Regarding risk factors for suicide, financial difficulties (33\%) and change of city to study at the university (45\%) were highlighted. There was an association between suicidal ideation with higher responsibilities (100\%) and pressure $(86 \%)$ in current life. This association was most significant between the history of suicide attempt $(\mathrm{p}=0.015)$ and depression $(\mathrm{p}=$ 0.010 ). Suicidal ideation was present in $12 \%$ of the sample.

CONCLUSION: In this study, it was found that part of the students presented suicidal ideation, while being exposed to risk factors that may contribute to develop or aggravate mental illness, reason that alerts the university management regarding the student's health care since the beginning admission to training.

\section{KEYWORDS: Nursing students; Prevention; Mental health; Suicide}

Submetido em 31-03-2019

Aceite em 18-11-2019

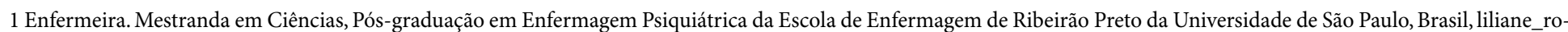
drigues23@hotmail.com

2 Enfermeira. Doutora em Ciências. Docente Assistente II na Universidade Federal de Mato Grosso, Campus Universitário do Araguaia. Brasil, alisseia@hotmail.com

3 Enfermeiro. Doutor em Bioética. Docente Adjunto da Universidade do Estado de Mato Grosso, Campus de Tangará da Serra, vagnernascimento@unemat.br

4 Enfermeira. Doutoranda em Enfermagem pela Universidade de São Paulo, Escola de Enfermagem, São Paulo, Brasil, rosamjvolpato@gamil.com

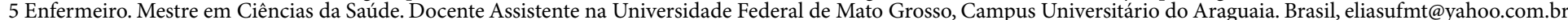

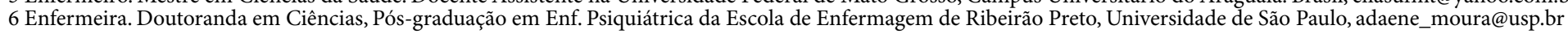




\section{INTRODUÇÃO}

A universidade é um local em que se desenvolve conhecimento científico, formação intelectual, profissional e moral, oportunizando ao jovem a possibilidade de assunção de seus atos e comportamentos, além de impor incertezas frente ao futuro (Matos, 2014).

Esse cenário gera ansiedade, preocupação e estresse, que somado à distância da família, frustações/perdas pessoais, cobranças, avaliações, reprovações, metodologias de ensino engessadas e tradicionais, pouco acolhimento de professores e gestão universitária, falta de incentivo e de políticas de permanência, vulnerabiliza o indivíduo ao sofrimento psíquico (Rios, Mascarenha, Souza, Olebar, Paiva, \& Silveira, 2019).

Pesquisadores revelam que tais enfrentamentos, com novos ciclos de amizade e relações afetivas, rearranjos quanto a alimentação/moradia/segurança e controle de recursos, exige habilidades sociais e competências emocionais nem sempre existentes entre os estudantes (Hirsch, Barlem, Almeida, Tomaschewski-Barlem, Lunardi, \& Ramos, 2018).

Entre os universitários, aqueles que cursam enfermagem, apesar de normalmente escolherem essa área por afinidade, e se satisfazerem ao longo da trajetória acadêmica, não estão imunes ao adoecimento (Silva, Silva, Chagas, Tortola, \& Caldeira, 2019a). O estranhamento diante da teoria e prática, e a excessiva cobrança de estar forte e preparado para atender/acolher todas as demandas da comunidade, especialmente em ser referência de cuidado desde o nascer ao morrer, trazem por vezes, respostas nocivas à saúde mental do estudante, como o constante questionamento sobre a vida e seus valores individuais, desencadeando em algumas circunstâncias ideação suicida (Silva, Nascimento, Santos, \& Ferreira, 2019b).

A partir disso, é indiscutível que o adoecimento mental desenvolvido durante a vida universitária, necessita de intervenção e acompanhamento profissional para reduzir prejuízos no desempenho do estudante e evitar riscos maiores, como àqueles ligados tanto à ideação suicida como ao suicídio propriamente dito (World Health Organization, 2014). Nesse sentido, este estudo teve como objetivo avaliar a presença de ideação suicida entre os estudantes de enfermagem e os fatores de risco associados com a presença da ideação suicida.

\section{MÉTODOS}

Trata-se de estudo exploratório, com abordagem quantitativa, realizada com estudantes da Universidade Federal de Mato Grosso (UFMT), Campus Universitário do Araguaia (CUA), localizado em Barra do Garças, Mato Grosso, Brasil.

Os critérios de inclusão foram: idade $\geq 18$ anos e estar regularmente matriculado no curso de enfermagem. Os critérios de exclusão foram: estudantes que não estiveram presentes na sala de aula no momento da coleta de dados ou que se encontraram afastados por atestado médico e licença de maternidade; e aqueles que deixaram de responder alguma das questões dos questionários aplicados, em específico do Inventário de Ideação Suicida de Beck (BSI).

Para a definição da amostra, utilizou-se uma amostragem não probabilística, por conveniência, dos 133 estudantes de enfermagem matriculados na UFMT/ CUA. Destes aceitaram participar do estudo 64 alunos. Houve perdas de quatro questionários, por preenchimento incompleto, totalizando assim, uma amostra de 60 universitários. Os estudantes autodeclararam pertencer ao $1^{\circ}$ semestre $(21 \%)$ do curso, seguido do $3^{\circ}$ semestre (32\%), $5^{\circ}$ semestre (20\%), $7^{\circ}$ semestre (22\%) e $8^{\circ}$ semestre (5\%).

A pesquisa foi realizada, nos períodos matutino e vespertino, entre os meses de julho a outubro de 2016, por meio da aplicação de três instrumentos de pesquisa. $\mathrm{O}$ primeiro, um questionário semiestruturado elaborado pelos pesquisadores, contendo questões objetivas, sobre os dados sociodemográficos (faixa etária, sexo, estado civil, renda individual mensal, religião e arranjo familiar) e os fatores de risco para o adoecimento mental. $\mathrm{O}$ segundo instrumento, o Inventário de Ideação Suicida de Beck (BSI), é um questionário validado no Brasil, autoaplicável que investiga a presença de ideação suicida, possuindo 21 itens. Obtém-se o escore total do BSI, somando os escores de cada item, pois, cada grupo apresenta alternativas, que podem ter escore de $0,1,2$, 3 , embora, este inventário não recomenda um ponto de corte. A presença de qualquer escore diferente de zero (0), em qualquer item, revela a existência de ideação suicida e demonstra a necessidade de o caso ser melhor investigado do ponto de vista clínico (Cunha, 2016). Os participantes responderam os instrumentos da pesquisa em sala de aula, após permissão do docente. $\mathrm{O}$ preenchimento teve a duração média de 30 minutos. 
Os dados foram lançados e organizados no programa Microsoft Office Excel 2013, posteriormente, foram processados e analisados no programa Epiinfo versão 3.5.2 e Bioestat versão 5.0. A estatística descritiva com utilização do programa Epi Info versão 3.5.2, foi aplicada nas variáveis sociodemográficas e nos fatores de risco, apresentados em gráficos e tabelas, por meio de números absolutos e relativos. Para avaliar as possíveis associações da ideação suicida com as demais variáveis (dificuldade financeira, morte significativa, mudança de cidade, pressão e responsabilidade na vida atual e solidão) foi utilizado o programa BioEstat versão 5.0, por meio da análise de correlação "Coeficiente de Contingência C" (resultado de $C=0$, determina que não há associação entre as variáveis, quando $C \neq 0$, há correlação entre duas variáveis ( $<0.1$ fraca, 0.1 a 0.3 moderada e $>0.3$ forte), sendo adotado o nível de significância de $0,05(\mathrm{p}<0,05)$.

Todas as providências em relação à dimensão ética do estudo foram tomadas de acordo com a Resolução de ética 466/2012. O estudo foi aprovado pelo Comitê de Ética em Pesquisa da Universidade Federal de Mato Grosso/Campus Universitário do Araguaia, sob o protocolo número 515/705. Todos os participantes do estudo manifestaram a sua concordância em participar do estudo, assinando o Termo de Consentimento Livre e Esclarecido (TCLE).

\section{RESULTADOS E DISCUSSÃO}

Os participantes do estudo apresentaram idade entre 18 e 37 anos, com média de 22 anos. Predominaram estudantes com idade entre 18 a 22 anos (72\%), do sexo feminino $(80 \%)$, solteiros $(82 \%)$, com renda mensal de até um salário mínimo (60\%), sem ocupação remunerada (80\%), com algum tipo de religião (83\%), residindo com o pai e/ou mãe (35\%) e sozinho (30\%) (Tabela 1).

Tabela 1 - Distribuição sociodemográfica dos estudantes de enfermagem da UFMT/CUA. Julho a outubro de 2016. Barra do Garças, MT, Brasil $(n=60)$.

\begin{tabular}{|l|l|c|c|}
\hline Descrição & & $\mathbf{N}$ & \% \\
\hline \multirow{4}{*}{ Faixa etária } & 18 a 22 & 43 & 72 \\
\cline { 2 - 4 } & 23 a 27 & 11 & 18 \\
\cline { 2 - 4 } & 28 a 32 & 04 & 07 \\
\cline { 2 - 4 } & 33 a 37 & 02 & 03 \\
\hline \multirow{2}{*}{ Total } & Feminino & 60 & 100 \\
\hline \multirow{2}{*}{ Sexo } & Masculino & 12 & 80 \\
\hline Total & 60 & 100 \\
\hline
\end{tabular}

\begin{tabular}{|c|c|c|c|}
\hline \multirow{4}{*}{ Estado civil } & Solteiro & 49 & 82 \\
\hline & Casado & 06 & 10 \\
\hline & União estável & 04 & 07 \\
\hline & Separado & 01 & 01 \\
\hline \multicolumn{2}{|l|}{ Total } & 60 & 100 \\
\hline \multirow{4}{*}{$\begin{array}{l}\text { Renda mensal indi- } \\
\text { vidual }\end{array}$} & $\begin{array}{l}\text { Até } 1 \text { salário } \\
\text { mínimo* }\end{array}$ & 36 & 60 \\
\hline & $\begin{array}{l}1 \text { a } 2 \text { salários } \\
\text { mínimos }\end{array}$ & 08 & 14 \\
\hline & $\begin{array}{l}\geq 3 \text { salários } \\
\text { mínimos }\end{array}$ & 02 & 03 \\
\hline & $\begin{array}{l}\text { Não declarou } \\
\text { renda }\end{array}$ & 14 & 23 \\
\hline \multicolumn{2}{|l|}{ Total } & 60 & 100 \\
\hline \multirow{2}{*}{ Ocupação remunerada } & Sim & 12 & 20 \\
\hline & Não & 48 & 80 \\
\hline \multicolumn{2}{|l|}{ Total } & 60 & 100 \\
\hline \multirow{2}{*}{ Possui Religião } & Sim & 50 & 83 \\
\hline & Não & 10 & 17 \\
\hline \multicolumn{2}{|l|}{ Total } & 60 & 100 \\
\hline \multirow{6}{*}{ Arranjo familiar } & Pai e/ou mãe & 21 & 35 \\
\hline & Sozinho & 18 & 30 \\
\hline & Com familiares & 10 & 17 \\
\hline & Amigos & 05 & 08 \\
\hline & Cônjuge & 03 & 05 \\
\hline & Em república & 03 & 05 \\
\hline \multicolumn{2}{|l|}{ Total } & 60 & 100 \\
\hline
\end{tabular}

*Salário mínimo correspondia a R\$ 880,00.

A partir dos resultados, verificou-se que as mulheres constituíram a maior parte da amostra, achado semelhante a um estudo realizado em Portugal, onde 64,7\% dos estudantes eram mulheres (Gonçalves, Sequeira, Duarte, \& Freitas, 2014) e em estudo brasileiro também realizado em Mato Grosso (53\%) (Santos, Marcon, Espinosa, Baptista, \& Paulo, 2017). Ambos os estudos, destacam a presença de mulheres em cursos da área da saúde, especialmente na enfermagem.

Neste estudo predominaram estudantes adultos jovens. Dados semelhantes foram encontrados em estudo realizado em Minas Gerais (BR) em que 59,1\% dos estudantes tinham idade entre 21 a 25 anos (Botti, Monteiro, Benjamim, \& Queiroz, 2016). Essa faixa etária pode estar relacionada ao perfil do curso, que comumente exige dedicação integral, sem ocupação com outras atividades (Coelho, Lorenzini, Suda, Rossini, \& Reimão, 2010). 
A maior parte dos estudantes eram solteiros (82\%), o que também foi verificado entre $92 \%$ dos estudantes de um estudo nordestino (BR) (Leão, Gomes, Ferreira, Cavalcante, 2018) e entre $72,6 \%$ dos estudantes do curso de graduação em Enfermagem do Rio Grande do Sul (BR) (Hirsch et al., 2018). Em relação a isso, alguns autores reportam que o estar solteiro pode favorecer o adoecimento mental, motivado pela ausência de companhia para conversar, desabafar, dividir sofrimentos e compartilhar experiências (Carlotto, Barcinski, Fonseca, 2015).

Parte dos participantes possuíam renda mensal de até um salário mínimo (60\%), assim como não exerciam atividade remunerada (80\%). Corroborando com a presente pesquisa, $60 \%$ dos estudantes de cursos da área da saúde de uma instituição pública em Mato Grosso (BR) referiram renda inferior a um salário mínimo (Mesquita, Lemes, Carrijo, Moura, Couto, Rocha, Volpato, 2016) e $80,7 \%$ dos universitários do Nordeste (BR) também não possuíam atividade remunerada (Leão, Gomes, Ferreira, Cavalcanti, 2018).

Uma associação da ideação suicida com a baixa condição socioeconômica foi reportada em estudo brasileiro, onde universitários com menos condições econômicas apresentaram maior probabilidade de ideação suicida ao compará-los aos universitários com níveis econômicos melhores (Santos et al., 2017). Uma revisão de literatura apontou que as condições econômicas têm sido relacionadas como fator de risco aos transtornos psicológicos, ao ponto que os estudantes que têm problemas financeiros tendem a evidenciar maiores níveis de depressão, ansiedade e ideação suicida (Pereira, Cardoso, 2015).

Em contrapartida, a OMS apontou que em países de rendimentos elevados, cerca de $90 \%$ das pessoas apresentavam adoecimento mental quando tentaram ou cometeram suicídio (WHO, 2014), aspecto que leva a reflexão da necessidade de prevenção ou tratamento em qualquer nível socioeconômico, para minimizar o adoecimento mental e consequentemente o comportamento suicida.

Grande parte dos estudantes do presente estudo autodeclararam professar religião (83\%), semelhante aos resultados identificados por Mesquita et al. (2016), onde professar uma religião foi reportado por $78 \%$ dos acadêmicos. Santos et al. (2017) ao comparar presença de ideação em acadêmicos com e sem práticas religiosas, identificaram que a presença de ideação suicida foi maior entre os acadêmicos que não possuíam religião.
Autores apontam que a religião é um importante fator que auxilia o estudante a superar dificuldades e estresse, próprios da fase acadêmica (Werner, Siqueira, Lemes, 2015).

Nesse estudo, observou-se que a maioria dos universitários reside com os pais (35\%) ou sozinhos (30\%). Morar com os pais foi semelhante em outras pesquisas nacionais realizadas em Mato Grosso (Mesquita et al., 2016), no Nordeste (Leão et al., 2018) e em um estudo internacional realizada em Portugal (Gonçalves, Sequeira, Duarte, \& Freitas, 2014). Diferente resultado foi apontado por Pereira e Cardoso (2015), onde os universitários moravam com colegas de classe $(66,4 \%)$ ou familiares $(23,8 \%)$.

\section{Fatores de risco para o adoecimento mental entre os estudantes de enfermagem}

$\mathrm{Na}$ tabela 2, verifica-se que 33\% dos universitários de enfermagem referiram passar por algum tipo de dificuldade financeira. Após o ingresso na universidade, 27\% dos acadêmicos enfrentam processo de luto de alguma pessoa importante (avós, pai e mãe e amigos) e $45 \%$ tiveram que mudar de cidade para frequentar o curso.

Tabela 2 - Fatores de risco para adoecimento mental entre os estudantes de enfermagem da UFMT/CUA. Julho a outubro de 2016. Barra do Garças, MT, Brasil $(n=60)$.

\begin{tabular}{|l|c|c|c|c|c|c|}
\hline Descrição & \multicolumn{2}{|c|}{ Sim } & \multicolumn{2}{c|}{ Não } & \multicolumn{2}{c|}{ Total } \\
\hline & $\mathrm{n}$ & $\%$ & $\mathrm{n}$ & $\%$ & $\mathrm{n}$ & $\%$ \\
\hline Dificuldade financeira & 20 & 33 & 40 & 67 & 60 & 100 \\
\hline $\begin{array}{l}\text { Morte de alguém im- } \\
\text { portante após entrar na } \\
\text { universidade }\end{array}$ & 16 & 27 & 44 & 73 & 60 & 100 \\
\hline $\begin{array}{l}\text { Mudança de cidade para } \\
\text { frequentar a universidade }\end{array}$ & 27 & 45 & 33 & 55 & 60 & 100 \\
\hline
\end{tabular}

Como observado, parte dos universitários referiram passar por algum tipo de dificuldade financeira, assim como apresentado no estudo de Santos et al. (2017). Pesquisa que objetivou estimar a prevalência e os fatores associados ao adoecimento mental entre estudantes universitários da área da saúde no Nordeste (BR), revelou maior prevalência do comprometimento da saúde mental entre os que apresentaram renda mensal de um a cinco salários mínimos (Leão et al., 2018).

Outro estudo aponta que as condições econômicas têm sido relacionadas como um fator associado aos transtornos psicológicos, entre eles a ideação suicida, desta forma, estudantes que têm problemas financeiros tendem a evidenciar maiores níveis de depressão, ansiedade e ideação suicida (Pereira, Cardoso, 2015). 
Essa relação foi confirmada em uma pesquisa brasileira que destacou associação da ideação suicida com a baixa condição socioeconômica, onde, universitários pertencentes a condições econômicas mais baixas apresentaram 1,69 mais chance a ideação suicida ao serem comparados aos demais universitários com níveis econômicos maiores (Santos et al., 2017).

Outro fator de risco verificado entre os estudantes foi a mudança de cidade. Neste estudo, parte dos acadêmicos (45\%) referiram ter que mudar de cidade para frequentar a universidade, fato que chama a atenção, pois, certamente esses estudantes tiveram que se (re)organizar em outras formas de moradia para conseguir frequentar a universidade. A necessidade dessa mudança também foi verificada em um estudo realizado em Portugal, onde, alguns alunos (119/349) precisaram deslocar-se de suas residências para frequentar a universidade, deixando a casa de seus familiares (Róias, 2016). Em contrapartida, um estudo realizado no Nordeste (BR) revelou que a maioria dos estudantes não mudaram de cidade para cursar a faculdade, pois mantiveram residindo com os pais ou cônjuge (Leão et al., 2018).

Para Pereira e Cardoso (2015, p. 24) como o "estudante universitário muitas vezes se afasta do seu local de residência para continuar a sua formação, pode-se notar quebra significativa nos contatos sociais e no número de pessoas disponíveis para obter apoio", desencadeando um conjunto de problemas mentais.

Os universitários que apresentaram ideação suicida declararam sentir maior pressão na vida atual (86\%) e maiores responsabilidades na vida hodierna (100\%) (tabela 3). Maior pressão na vida do universitário também foi verificada no estudo de Sequeira, Carvalho e Borges (2013), em que 69,7\% dos acadêmicos de enfermagem relataram sentirem-se sob grande pressão e estresse. Já um estudo paulista (BR) identificou certa exigência emocional, principalmente nos períodos de estágio entre estudantes de psicologia de uma universidade pública (Andrade, Antunes, Zanoto, Tiraboschi, Viana, Currila, 2016).

Para Robazzi (2019), o contexto acadêmico associado à pressão e maiores responsabilidades adquiridas, desperta no estudante uma fase de concorrência, competitividade em busca dos melhores resultados e melhor possibilidade de empregos após a formação. Silva (2013) aponta em seu estudo, que ao ingressar na universidade o indivíduo se depara com várias situações estressantes, tais como: excesso de conteúdo, noites mal dormidas, baixa qualidade de vida, desentendimento entre professor e aluno, entre outras.
Entre os fatores de risco, a solidão esteve presente entre $100 \%$ dos universitários que apresentaram ideação suicida. Os resultados apontam evidências para uma forte associação da ideação suicida com a solidão entre os estudantes $(\mathrm{C}=0,321 ; \mathrm{p}=0,025)$ (tabela 3$)$. Corroborando com os dados apresentados, o estudo de Sequeira, Carvalho e Borges (2013) revelou que 43,6\% dos estudantes de enfermagem referiram solidão no último mês que antecedeu a pesquisa.

Tabela 3 - Associação da presença de ideação suicida dos estudantes de enfermagem da UFMT/CUA com fatores de risco. Julho a outubro de 2016. Barra do Garças, MT, Brasil, ( $n=60)$.

\begin{tabular}{|c|c|c|c|c|}
\hline \multirow{3}{*}{ Variáveis } & \multicolumn{2}{|c|}{$\begin{array}{c}\text { Presença de ideação } \\
\text { suicida }\end{array}$} & \multirow{2}{*}{\multicolumn{2}{|c|}{ Estatística }} \\
\hline & \multirow{2}{*}{$\frac{\text { Sim }}{\mathrm{N}(\%)}$} & \multirow{2}{*}{$\frac{\text { Não }}{\mathrm{N}(\%)}$} & & \\
\hline & & & $\begin{array}{c}\text { Teste de } \\
\text { Contingência }\end{array}$ & $\begin{array}{l}\text { Valor } \\
\text { de p }\end{array}$ \\
\hline \multicolumn{5}{|c|}{ Maior pressão na vida atual } \\
\hline Sim & $06(86 \%)$ & $49(92 \%)$ & 0,078 & 0,903 \\
\hline Não & $01(14 \%)$ & $04(8 \%)$ & & \\
\hline \multicolumn{5}{|c|}{ Maior responsabilidade na vida atual } \\
\hline Sim & $07(100 \%)$ & $50(94 \%)$ & 0,083 & 0,781 \\
\hline Não & $00(0 \%)$ & $03(6 \%)$ & & \\
\hline \multicolumn{5}{|l|}{ Solidão } \\
\hline Sim & $07(100 \%)$ & $25(47 \%)$ & 0,321 & $0,025^{\star}$ \\
\hline Não & $00(0 \%)$ & $28(53 \%)$ & & \\
\hline \multicolumn{5}{|c|}{ Histórico de tentativa de suicídio } \\
\hline Sim & $03(50 \%)$ & $04(7 \%)$ & 0,369 & $0,015^{\star}$ \\
\hline Não & $03(50 \%)$ & $50(93 \%)$ & & \\
\hline \multicolumn{5}{|c|}{ Histórico de depressão na vida } \\
\hline Sim & $05(71 \%)$ & $10(19 \%)$ & 0,363 & $0,010^{*}$ \\
\hline Não & $02(29 \%)$ & $43(81 \%)$ & & \\
\hline
\end{tabular}

${ }^{\star} \mathrm{p}=<0,05$

É importante ressaltar que a solidão, pode estar relacionada a uma baixa rede de suporte social, o que contribui para a piora da saúde mental dos estudantes, assim como relatado no estudo de Arria, O'Grady, Caldeira, Vincent, Wilcox, \& Wish (2009), onde a carência de suporte social entre os estudantes é um fator de risco à ideação suicida. A carência expressada pelo sentimento de solidão está relacionada com a satisfação com a vida. Maiores níveis de solidão estão diretamente associados a um aumento da ideação suicida (Nascimento, Morales, 2016). O estudo de Krause-Parello (2008) confirma essa necessidade, ao ressaltar que estudantes que precisam ausentar do seio da sua família podem desencadear sentimentos negativos perigosos para o adoecimento mental, por sentirem-se emocionalmente isolados e sozinhos nesta nova fase da vida. 
No presente estudo, verificou-se que $12 \%$ dos universitários apresentaram histórico de tentativa de suicida e $25 \%$ histórico para depressão. Além disso, houve uma forte associação entre o histórico de depressão e de tentativa de suicídio com a presença de ideação suicida entre os estudantes $(C=0,363 / p=0,010 ; C=0,369$ / $\mathrm{p}=0,015$ ) (tabela 3). Semelhantemente ao que aqui foi apresentado, pesquisa apontou que $28,6 \%$ dos estudantes mencionaram episódios depressivos na vida (Leão et al., 2018), enquanto que entre estudantes mato-grossense a depressão esteve presente em $41 \%$ da amostra (Mesquita et al., 2016).

Quanto à ideação suicida, um estudo mineiro (BR) revelou que 9,5\% dos alunos reportaram histórico de tentativa de suicídio. Já em um estudo mato-grossense (BR) 9\% dos universitários reportaram tentativa de suicídio, porém, na perspectiva dos familiares, apontando ainda, uma acentuada associação com a ideação suicida entre essas tentativas (Santos et al., 2017).

Acima do que foi encontrado nesta pesquisa, o estudo de Veloso, Lima, Sales, Monteiro, Gonçalves, Silva Junior (2019) realizado no Piauí (BR) entre universitários da área da saúde, revelou que $21 \%$ dos estudantes apresentaram histórico de tentativa de suicídio e que possuir histórico de tentativa de suicídio aumenta em 243 vezes as chances de ideação suicida ( $\mathrm{IC}=51,308$ 1150,874). Santos et al. (2017), revelaram em seu estudo que possuir histórico de suicídio na família ou entre amigos próximos contribui para aumentar a propensão a apresentar ideação suicida entre os universitários.

A Organização Mundial da Saúde aponta como fator de risco para o suicídio, a ideação suicida e o histórico de tentativa de suicídio (WHO, 2014) e confirmando essa afirmação, na Colômbia, a probabilidade de ideação suicida foi de 4,4 vezes naqueles que fizeram uma tentativa de suicídio, do que naqueles que não o fizeram (Cuesta, Parra, Orozco, \& Pérez, 2015).

Como apontado, o adoecimento mental esteve presente em parte dos estudantes que apresentaram ideação suicida, em específico a depressão, dados semelhantes a estudo internacional (Róias, 2016). O estudo de Santos et al. (2017) revelou que a variável sintomas depressivos têm uma acentuada associação com a ideação suicida $(\mathrm{p}<0,001)$.

\section{Ideação suicida entre acadêmicos de enfermagem}

No gráfico 1, pode-se verificar que a ideação suicida esteve presente em $12 \%$ dos acadêmicos.
Outros estudos apontaram a presença de ideação suicida entre os estudantes semelhante aos dados aqui apresentados, como um estudo realizado em Portugal (72,6\%) (Róias, 2016), na Colômbia (16\%) (Cuesta et al., 2015), e no Irão (11,7\%) (Poorolalal, Panahi, Ghaleiha, Jalili \& Darvishi, 2017).

Gráfico 1 - Presença de ideação suicida entre os acadêmicos de enfermagem da UFMT/CUA. Julho a outubro de 2016. Barra do Garças, MT, Brasil, $(n=60)$.

Ideação suicida entre os acadêmicos de enfermagem

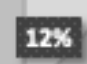

口COMIDEAÇÃ̄O 口SEMIDEAÇূ̃o

$88 \%$

Para Botti, Monteiro, Benjamim, \& Queiroz (2016) independente da expressão numérica ser relevante ou não para apontar o risco do suicídio na vida do estudante, é preciso atentar a presença de adoecimento mental entre os universitários, uma vez que, é mais comum nessa nova fase de vida se deparar com alguns empasses que entravam sua saúde mental.

Para Róias (2016) a presença de ideação suicida entre os estudantes precisa ser levada muito a sério, pois tem sido considerado forte indicador de futuro suicídio, e desta forma, precisa da atenção redobrada dos profissionais de saúde. Portanto, há necessidade de desenvolver de forma conjunta atividades preventivas e de intervenção breve.

Assim, a instituição de ensino superior precisa se (re) organizar no sentido de desenvolver programas que visem a promoção da saúde mental e a prevenção do suicídio entre os universitários, de forma que possibilite no âmbito da academia, espaços abertos para debates acerca da temática, buscando reduzir tabus que cercam o tema e disseminar o assunto por todos do espaço acadêmico (Gonçalves, 2014).

Pereira (2013) ressalta a importância de preparar os estudantes para enfrentar todas as adversidades da vida acadêmica, em específico os da saúde. Desta forma, o despertar dos alunos quanto aos aspectos preventivos desse adoecimento deve ocorrer o mais breve, de maneira que possam enfrentar com mais entusiasmo o cotidiano acadêmico. 
Esse enfrentamento pode ser baseado em grupos, disponibilizando salas de apoio psicopedagógico e/ou através de programas de saúde e bem estar universitário, reduzindo as vulnerabilidades para o adoecimento e danos mentais (Sequeira, Carvalho, Borge, 2013).

\section{CONCLUSÃO}

Neste estudo, integraram-se predominantemente jovens universitários, do sexo feminino, solteiros, com religião, baixa renda mensal e residindo sozinho ou com os pais. Verificou-se que os estudantes apresentaram fatores de risco que podem contribuir com o adoecimento mental, entre eles, dificuldade financeira e a necessidade de mudar de cidade para cursar a universidade. Parte dos estudantes de enfermagem apresentaram ideação suicida, destacando que as variáveis que apresentaram associação com a ideação suicida foram a solidão, o histórico de tentativa de suicídio e o histórico de depressão.

Os resultados apresentaram aspectos situacionais dos estudantes de enfermagem, despertando para a necessidade da criação de ambientes que acolham, acompanhem e rastreiem regularmente a presença de qualquer sofrimento mental, em busca de reduzir os prejuízos reais e potenciais.

Acredita-se que a criação de espaços individuais ou coletivos facilitará a realização de ações de prevenção e promoção a saúde desses estudantes, podendo contribuir para melhoria da qualidade de vida.

Diante do exposto, acredita-se que todo os cuidados propostos voltados a cuidar da saúde mental dos estudantes possam contribuir para que estes reconheçam que necessitam de ajuda o mais precoce possível e encontrem condições/referências para receberem atendimento e acompanhamento no âmbito universitário.

\section{IMPLICAÇÕES PARA A PRÁTICA CLÍNICA}

O presente estudo poderá contribuir com a sensibilização dos reitores, pró-reitores, coordenadores de cursos, professores e servidores de programas de assistência estudantil, responsáveis por elaborar políticas e estratégias que visem a prevenção, diagnóstico e tratamento precoce em busca de acolher os estudantes no que diz respeito ao seu sofrimento mental.
Além de acolher e atender as necessidades dos universitários, tais ações (prevenção e promoção) contribuirá para a desmistificação dos temas que envolvem a saúde mental, em especial ao suicídio, favorecendo assim um processo de discussão, aproximando os estudantes, na identificação de suas necessidades e como mediá-la a partir do suporte profissional e de serviços especializados presente na comunidade.

\section{REFERÊNCIAS BIBLIOGRÁFICAS}

Andrade, A. S., Antunes, N. A., Zanoto, P. A., Tiraboschi, G. A., Viana, P. V. B. A., Currila, R. T. (2016). Vivências Acadêmicas e Sofrimento Psíquico de Estudantes de Psicologia. Psicologia: Ciência e Profissão, 36(4): 831846. Doi: 10.1590/1982-3703004142015

Arria, A. M., O'Grady, K. E., Caldeira, K. M., Vincent, K. B., Wilcox, H. C., \& Wish, E. D. (2009). Suicide ideation among college students: A multivariate analysis. Archives of Suicide Research.,13 (3):230-246. Doi: 10.1080/13811110903044351

Botti, N. C. L., Monteiro, A. M. C., Benjamim, M. L. N., \& Queiroz, L. C. (2016). Depressão, uso de drogas, ideação e tentativa de suicídio entre estudantes de enfermagem. Revista de Enfermagem UFPE. 10 (7):2611-2616. Disponível em: https://periodicos.ufpe.br/revistas/revistaenfermagem/article/view/11321/13010

Carlotto, M. S., Barcinski, M., Fonseca, R. (2015). Transtornos mentais comuns e associação com variáveis sociodemográficas e estressores ocupacionais: uma análise de gênero. Estudos e Pesquisas em Psicologia, 15(3): 1006-1026. Disponível em: http://pepsic.bvsalud.org/scielo.php?script=sci_abstract\&pid $=$ S1808-42812015000300013

Coelho, A. T., Lorenzini, L. M., Suda, E. Y., Rossini, S., \& Reimão, R. (2010). Qualidade de sono, depressão e ansiedade em universitários dos últimos semestres de cursos da área da saúde. Neurobiologia, 73:35-39. Disponível em: https:/www.researchgate.net/profile/Rubens_Reimao/publication/236943130_Sleep_ quality_depression_and_anxiety_in_college_students_of_last_semesters_in_health_areas_courses/ links/0deec51a4a98ad9d76000000.pdf 
Cuesta, O. M. B., Parra, J. A. C., Orozco, M.Z. M., \& Pérez, O. A. M. (2015). Ideación suicida y factores asociados en jóvenes universitarios de la ciudad de Medellín. Revista Archivo Médico de Camagüey, 19(5):469-478. Disponível em: http://scielo.sld.cu/scielo.php?script=sci_ arttext\&pid=S1025-02552015000500006\&lng=es\&tlng=es

Cunha, J. A (2016). Manual da versão em português das Escalas Beck. São Paulo: Casa do Psicólogo Livraria e Editora.

Gonçalves, A. M., Sequeira, C., Duarte, J. C., \& Freitas, P. P. (2014). Ideação Suicida em Estudantes do Ensino Superior Politécnico: Influência de Algumas Variáveis Sociodemográficas, Académicas e Comportamentais. Millenium, 47: 191203. Disponível em: https://www.ipv. pt/millenium/Millenium47/16.pdf

Hirsch, C. D., Barlem, E. L. D., Almeida, L. K., Tomaschewski-Barlem, J. G., Lunardi, V. L., \& Ramos, A. M. (2018). Fatores percebidos pelos acadêmicos de enfermagem como desencadeadores do estresse no ambiente formativo. Texto e Contexto Enfermagem, 27(1). Doi: 10.1590/0104-07072018000370014

Krause-Parello, C. A. (2008). Loneliness in the School Setting. The Journal of School Nursing. 24 (2): 66-70. Doi: $\quad$ 10.1622/1059-8405(2008)024[0066:LITSS]2.0. $\mathrm{CO} ; 2$

Leão, A. M., Gomes, I. P., Ferreira, M. J. M., Cavalcanti, L. P. G. (2018). Prevalência e Fatores Associados à Depressão e Ansiedade entre Estudantes Universitários da Área da Saúde de um Grande Centro Urbano do Nordeste do Brasil. Revista Brasileira de Educação Médica, 42(4): 55-65. Doi: 10.1590/1981-52712015v42n$4 \mathrm{rb} 20180092$

Matos, N.A. (2014). Conhecendo o sofrimento psíquico dos universitários da Faculdade de Ceilândia. (Monografia). Universidade de Brasília, Brasil. Disponível em: https://bdm.unb.br/handle/10483/6906

Mesquita, A. M., Lemes, A. G., Carrijo, M. V. N., Moura, A. A. M., Couto, D. S., Rocha, E. M., Volpato, R. J. (2016). Depressão entre estudantes de cursos da área da saúde de uma Universidade em Mato Grosso. Journal Health NPEPS, 1(2): 218-230. Disponível em: https://periodicos.unemat.br/index.php/jhnpeps/article/view/1433
Nascimento, I. M. C.; Morales, D. R. D. (2016). Comportamento suicida em estudantes de enfermagem do Campus Senador Helvídio Nunes de Barros; Universidade Federal do Piauí. ACADEMO Revista de Investigación en Ciencias Sociales y Humanidades. 3 (1). Disponível em: http://revistacientifica.uamericana.edu.py/ index.php/academo/article/view/33

Pereira, A. G.; Cardoso, F. S. (2015). Ideação suicida na população universitária: uma revisão de literatura. Revista Eletrónica de Psicologia, Educação e Saúde. 5 (2):16-34. Disponível em: https://revistaepsi.com/wpcontent/uploads/artigos/2015/Ano5-Volume2-Artigo2.pdf

Poorolalal, J., Panahi, S., Ghaleiha, A., Jalili, E., \& Darvishi, N. (2017). Suicide and Associated Risk Factors Among College Students. International Journal of Epidemiologic Research, 4(4): 245-250. Disponível em http://ijer.skums.ac.ir/article_28778_5d5e51502d35e2 9a1293ac8b6b136043.pdf

Rios, M. G. V., Mascarenha, L. V. R., Souza, K. S., Olebar, D. T. C. R., Paiva, M. C. E. \& Silveira, A. O. (2019). Adoecimento e sofrimento psíquico entre universitários: estado da arte. Revista Humanidades e Inovação, 6(8), 2331. Disponível em: https://revista.unitins.br/index.php/ humanidadeseinovacao/article/view/1259

Robazzi, M. L. C. C. (2019). Promoção da saúde física e mental e de bem estar no ambiente universitário. SMAD. Revista eletrônica saúde mental álcool e drogas, 15(2), 1-3. Doi: 10.11606/issn.1806-6976.smad.2019.154951

Róias, C. P. C. (2016). Autodano e ideação suicida na população estudantil da Universidade dos Açores. (Dissertação de Mestrado). Departamento de Ciências da Educação, Universidade dos Açores, Portugal. Disponível em: https://repositorio.uac.pt/ bitstream/10400.3/3782/1/DissertMestradoCarlaPatriciaCostaRoias2016.pdf

Santos, H. G. B., Marcon, S. R., Espinosa, M. M., Baptista, M. N., \& Paulo, P. M. C. (2017). Fatores associados à presença de ideação suicida entre universitários. Revista Latino-Americana de Enfermagem, 25(15), 1-8. Doi: 10.1590/1518-8345.1592.2878 
Sequeira, C. A., Carvalho, J. C., \& Borges, E. M. (2013) Vulnerabilidade mental em estudantes de enfermagem no ensino superior: estudo exploratório. Journal of Nursing and Health. 3 (2):170-181. Doi: 10.15210/ jonah.v3i2.3551

Silva, C. K. (2013). Prevalência de sintomas de ansiedade e depressão entre os acadêmicos da Faculdade de Medicina da Bahia. (Monografia). Faculdade de Medicina - Universidade Federal da Bahia, Brasil. Dsponível em: https://repositorio.ufba.br/ri/handle/ ri/13966? mode $=$ full

Silva, P. L. B. C., Silva, B. F. F., Chagas, K. K. A. C. R., Tortola, M. B. A., \& Caldeira R. L. R. (2019). Transtorno Mental comum entre Estudantes de Enfermagem e Fatores Envolvidos. Revista de Enfermagem do Centro Oeste Mineiro, 9, e3191.Doi: 10.19175/recom.v9i0.3191

Silva, R. G. M., Nascimento, V. F., Santos, P. O. F., \& Ferreira M. Z. J. (2019). Teste de Papanicolau: realização e conhecimento de acadêmicas de enfermagem. Revista de Epidemiologia e Controle de Infecção, 9(1), 81-86. Doi: 10.17058/reci.v9i1.11592
Veloso, L. U. P., Lima, C. L. S., Sales, J. C. S., Monteiro, C. F. S., Gonçalves, A.M.S., Silva Junior, F. J.G. (2019). Ideação suicida em universitários da área da saúde: prevalência e fatores associados. Revista Gaúcha de Enfermagem, 40, 1-6. Doi: 10.1590/1983- 1447.2019.20180144

Werner, M. E. C., Siqueira, M. F. C., Lemes, A. G. (2015). Consumo de álcool entre universitários. Vamos discutir essa ideia? Interdisciplinar: Revista Eletrônica da UNIVAR.1 (13), 42-48. Disponível em: https://www. researchgate.net/publication/322527332_Consumo_ alcoolico_entre_universitarios_Vamos_discutir_essa_ ideia/download

World Health Organization. (2014). Preventing suicide: a global imperative. Disponível em: http://apps.who. int/iris/bitstream/10665/131056/1/9789241564779_ eng.pdf 